\title{
AeroDAQ: A Workspace based application
}

\author{
$\underline{\text { N. Sankaranarayanan }}^{\mathrm{a}}$ and R. S. Humphries ${ }^{\mathrm{b}}$ \\ ${ }^{a}$ CSIRO Data61, Clayton VIC Australia and ${ }^{b}$ CSIRO Climate Science Centre, O\&A, Aspendale VIC Australia \\ Email: Nirupama.Sankaranarayanan@data61.csiro.au
}

\begin{abstract}
AeroDAQ is an original, stand-alone, Workspace-based software application developed by the CSIRO that provides a robust, open, flexible, and reliable data acquisition and visualisation system for Condensation Particle Counters (CPCs). These instruments are fundamental to air quality work and ongoing atmospheric composition measurements around the globe such as those aboard the highly publicised RV Investigator and Cape Grim Baseline Air Pollution Station.
\end{abstract}

The original software that comes with the instrument has several key limitations. The software is unable to run reliably for more than a few days and thereby requires regular manual restarting of the application. Additionally, data is saved in a proprietary binary format unable to be read outside the software environment provided by the vendor without export. Data visualisation in the software is also limited, restricting the ability to review data and performance in real-time. AeroDAQ was born of a desire to have a lightweight, portable, and reliable application that manages data acquisition from the instrument and data visualisation.

The Workspace scientific workflow and application development platform is well suited to the development of a stand-alone software application - AeroDAQ in this case. Workspace provides a versatile and extensible plugin architecture and has a close relationship to the Qt Toolkit. Workspace provided an easy interface for implementing both a serial port plugin for communication with the CPC and an application to capture and store received data. The vast suite of built-in operations allowed for quick creation of the complex workflows required to capture, process, and display data from the CPC. Third-party charting libraries like NVD3, VisJs and PlotLy are wrapped into Workspace and with the impressive range of built-in widgets, visualisation of data was made easy.

AeroDAQ represents the adoption of a new experimental standard in data acquisition and storage from CPC devices. It increases the productivity of the research scientists and technicians by providing them with the ability to visualise their data in real-time, allowing them to quickly identify and rectify instrument issues and experimentally respond to the scientific observations where appropriate. This reduces the research team's downtime, opens the data for presentation to others, increases confidence in the quality of the collected data, and enhances scientific output.

Keywords: Workspace, extensible architecture, visualisation 


\section{INTRODUCTION}

Condensation Particle Counters (CPCs) are scientific instruments used by researchers across the globe to count atmospheric aerosol particles in a range of environments and applications. This includes: laboratory setups, sensitive environments (schools, workplaces), vehicle emissions, pharmaceutical cleanrooms or in the ambient atmosphere to monitor atmospheric composition relevant for air quality and climate studies. Aerosol is a major component of poor air quality (otherwise referred to as particulate matter), and CPCs are a core measurement device quantifying the air quality. CSIRO's Climate Science Centre regularly deploys CPCs to measure ambient atmospheric composition during intensive experimental field campaigns and during long-term monitoring programs such as those aboard the RV Investigator and at Cape Grim Baseline Air Pollution Station. A CPC makes real-time measurements of atmospheric particles larger than $3 \mathrm{~nm}$ by growing them to sizes where they can be counted optically. The original software that comes with the instrument has limitations which can significantly impact data analysis. Additionally, data is saved in a proprietary binary format unable to be read outside the software environment provided by the vendor except after laborious manual exporting. This slows down data analysis. Parameters that indicate instrument health are also difficult to access from the software, creating scenarios where data quality can suffer without the user's knowledge, particularly in scenarios where the instrument is operated remotely. AeroDAQ - a lightweight, portable, and reliable application - was developed by CSIRO Data61 in collaboration with CSIRO Climate Science Centre scientists, to better manage data acquisition from the $\mathrm{CPC}$, and to provide real-time visualisation of both aerosol and instrument parameter data.

\section{OVERVIEW OF SOFTWARE DEVELOPMENT USING WORKSPACE}

AeroDAQ was developed using CSIRO Workspace (Bolger et al., 2015, Watkins et al., 2017; Cleary et al., 2017), a cross-platform workflow and application development framework designed to enhance productivity, enable collaboration and software reuse, and to enable the commercialisation of software products. AeroDAQ is a Workspace-based software application like Dive Mechanic (R. C. Z. Cohen et al., 2017) and ArcWeld (Anthony B. Murphy et al., 2017).

A Workspace workflow is a series of operations connected by directed data flows and order of execution. Workspace offers a vast suite of built-in operations which reduces the development effort. The easily extendible plugin architecture of Workspace makes it an ideal choice for applications like AeroDAQ which need integration with a third-party charting library (PlotLy) and the development of a serial port interface. Workflows can easily be combined with a graphical user interfaces (GUIs) to be deployed as stand-alone compiled applications. The fundamental requirements of AeroDAQ are -

1. Interface to the instrument via the available interface port - a serial port

2. Read the data output from the instrument every second

3. Parse the data output, which is formatted as a string

4. Format the received data such that it is:

a. written to a non-proprietary readable format e.g., text, CSV files and a simple database

b. timestamped with local time

c. plotted in real-time i.e., as soon as it is received from $\mathrm{CPC}$, with the ability to explore data interactively

5. House a built-in command-line interface in the application, where commands can be sent to the instrument.

6. Collect instrument status every 15 minutes

7. Decouple the acquisition process from the display routines by making the data acquisition and storage workflow independent of the visualisation workflow

8. Incorporate timestamps in the file names of stored data files for ease of retrospective analysis

9. Save metadata directly within the data files to ensure data provenance

10. Cycle stored database files daily to facilitate ease of retrospective analysis and ensure against data corruption

11. Cycle stored raw data and CSV files every 3 hours to facilitate ease of retrospective analysis and ensure against data corruption 
12. Store and display data with minimal to no human intervention (once the application has been started it is expected to run for several days/weeks)

13. Automatically recover data acquisition if the serial cable is disconnected from the CPC and subsequently re-connected

\section{AeroDAQ DEVELOPMENT USING WORKSPACE}

AeroDAQ is comprised of 2 main applications:

- AeroDAQ data collector - interfaces to the instrument via a serial port facilitates sending commands to the $\mathrm{CPC}$ and reads, parses, and stores the received data

- AeroDAQ visualiser - visualises the received sample data and instrument status in the form of userinteractive tables and charts

\subsection{AeroDAQ data collector}

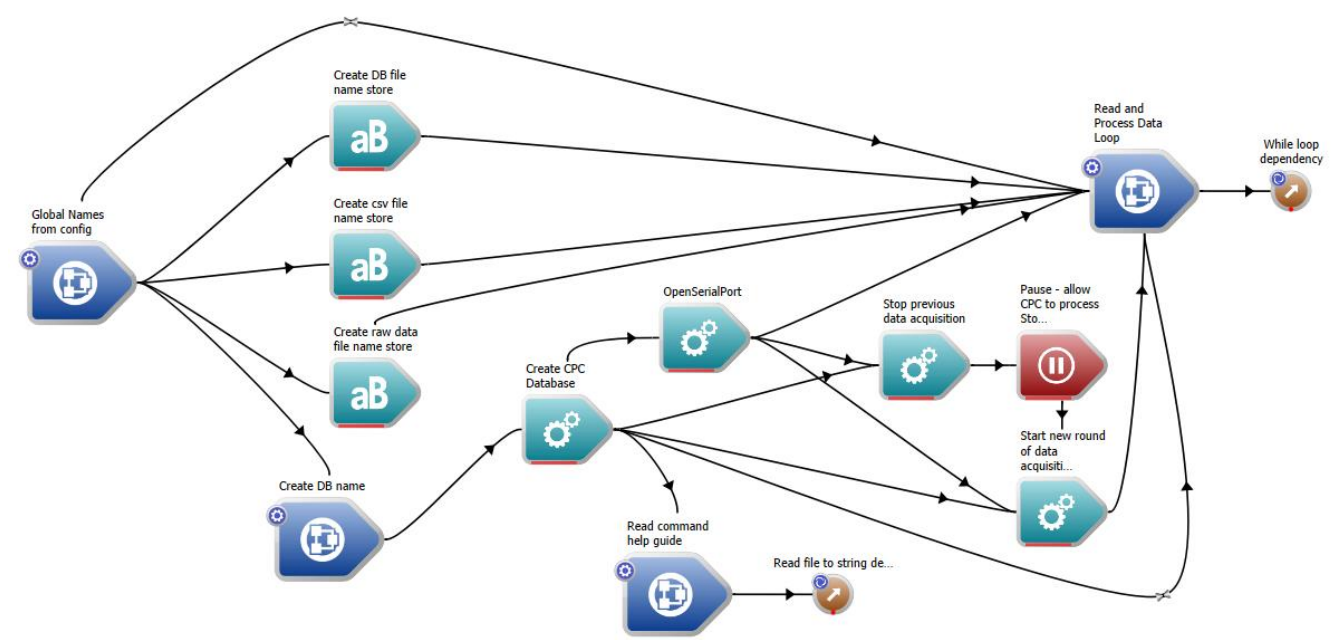

Figure 1. AeroDAQ data collector Root workflow

Figure 1 shows the Root workflow of the AeroDAQ data collector application. The workflow reads the configuration file which stores meta-data including information on which COM port to use. It then creates the database, CSV and text files used to store received data, instrument status and any commands written to the $\mathrm{CPC}$. The workflow provides the user with access to write a command to the CPC as needed. It then runs an infinite loop of reading, processing, and storing data received from the CPC. This "Read and Process Data Loop" is a nested workspace in the Root workflow and is depicted in Figure 2.

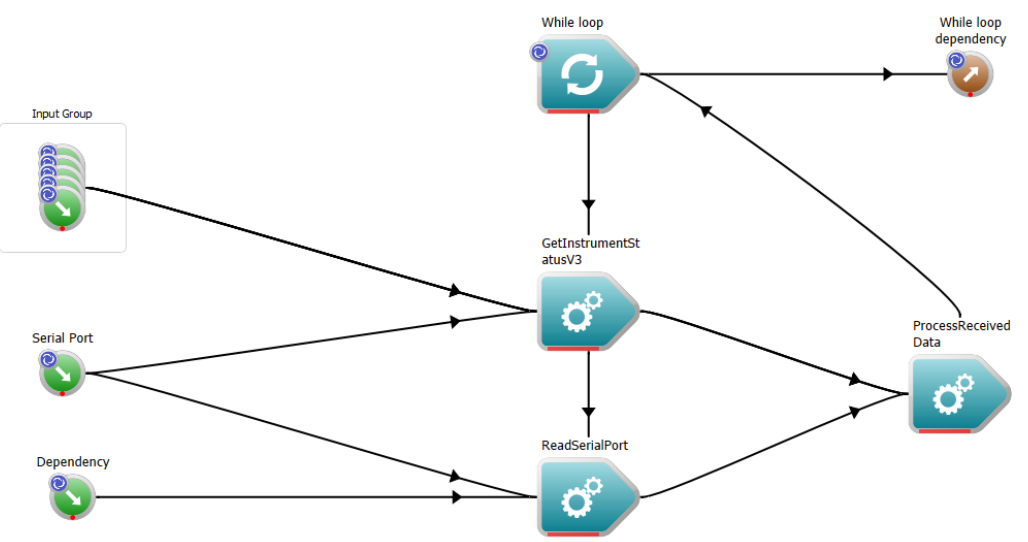

Figure 2. Read and process data loop 
The data collector captures sample data sent from the CPC. The CPC outputs data once/second. An example of data received from the CPC is given below -

$U 1,1405,1327,1404,1420,1370,1429,1403,1369,1364,1406,8.43 E+02,7.96 E+02,8.43 E+02,8.52 E+02,8.22 E+0$ $2,8.57 E+02,8.42 E+02,8.21 E+02,8.18 E+02,8.44 E+02,1,0.00,0.00,0$

The data collector captures and parses the received string, averaging out the $10 \mathrm{~Hz}$ concentration values and timestamping the received data to make it easier for retrospective analysis by a human user (in lieu of using the arbitrary UX value). Data collection is paused by the workflow (seen in Figure 2) every 15 minutes while the instrument status is read. The operation "GetInstrumentStatus" keeps track of how long it has been since the last instrument status report.

An example of instrument status received from the $\mathrm{CPC}$ is given below -

9.67E+02,0000,38.9,22.0,40.1,36.6,101.5,70.0,2.696,25,FULL (2469)

The UI, as seen in Figure 3, offers a simple interface to start/stop data collection by writing a command to the CPC, view received data and write to the CPC. A comprehensive command help guide is also provided.

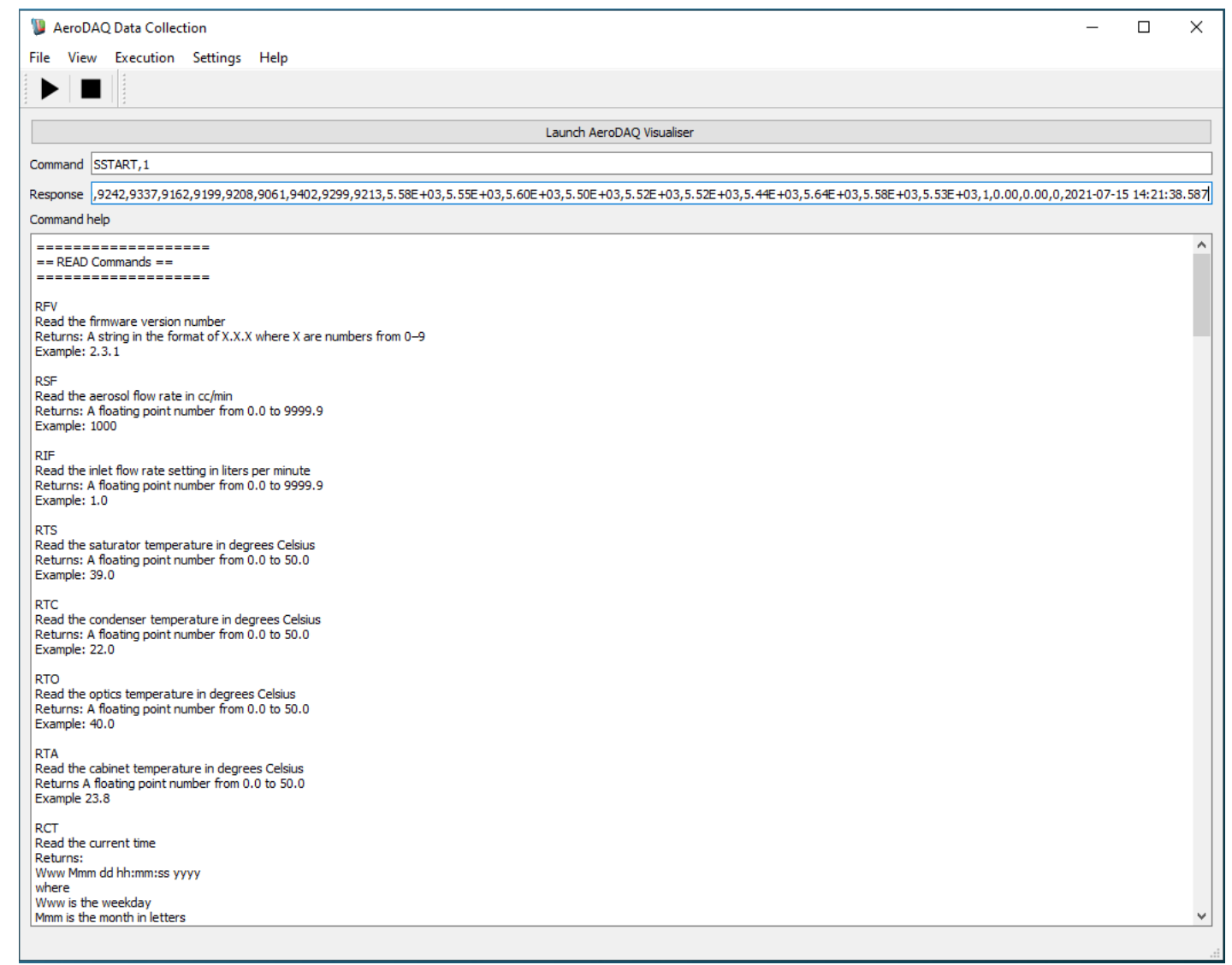

Figure 3. AeroDAQ Data Collection application UI

\subsection{AeroDAQ visualiser}

The AeroDAQ visualiser provides the user with dynamic reports on both the collected data and current instrument status. The data and instrument status reports are provided to the user in the form of tables and charts so that the user can immediately determine the status of the instrument and the data collected. This is critical to enable the scientist/technician to ensure the proper functioning of the instrument, any temporal deterioration in instrument performance, or to identify and react in real-time to the observations being made or experiments being conducted. The visualiser reads the data recorded in the database by the AeroDAQ data collector.

Figure 4 shows us the workflow responsible for reading the recorded concentration and analog values and plotting them. 


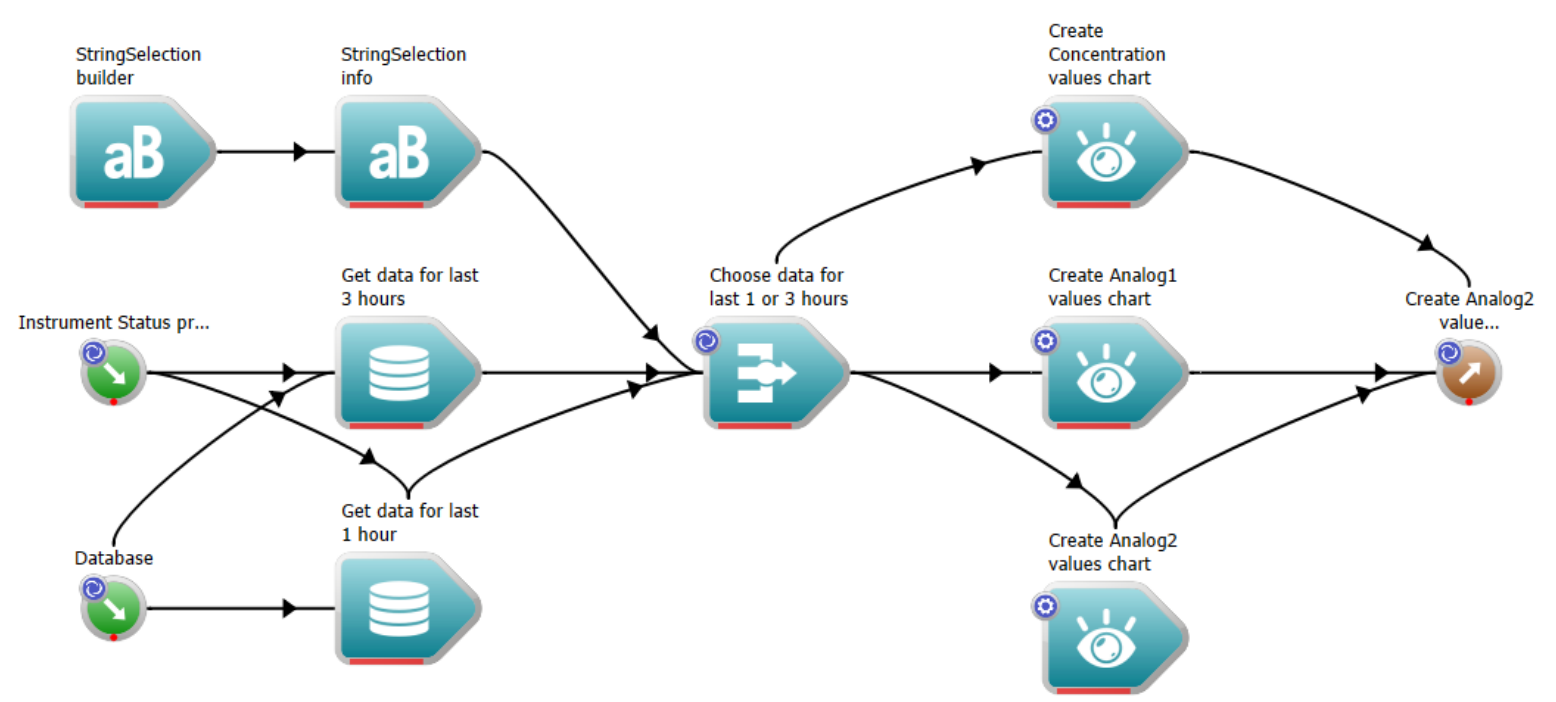

Figure 4. Reads concentration and analog values and create charts

Figure 5 shows the workflow that retrieves the instrument status from the database and plots the various instrument status values such as saturation temperature, condenser temperature, optics temperature, cabinet temperature, ambient pressure, orifice pressure, nozzle pressure.

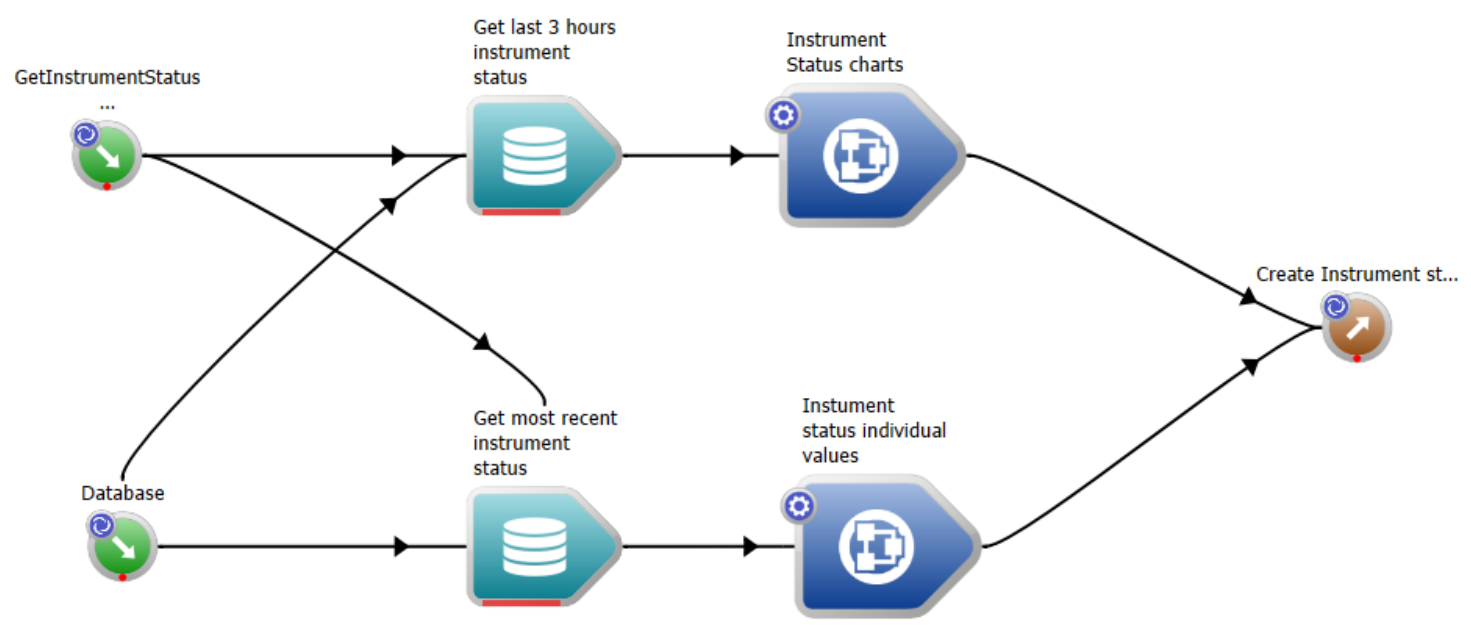

Figure 5. Root workflow to plot instrument status charts

\section{RESULTS}

A major international experimental campaign occurred just south of Sydney, Australia during the 2019/20 summer, the aim of which was to characterise the aerosol formation from emissions from eucalyptus forests. The summer came to be known colloquially known as Black Summer because of the anomalously intense bushfires that occurred throughout the country. In response to the state of emergency, the campaign's focus was redirected to capturing the impact of bushfire smoke on the air quality and the subsequent health implications on the Australian population.

A CPC (TSI model 3776) was deployed as part of the campaign and AeroDAQ was chosen as the data acquisition system for the second half of the campaign. The software ran continuously, remaining stable and performing to specification throughout the 30-day deployment.

Figure 6 shows the chart of concentration of particulate matter recorded per second for the previous 3 hours, providing the scientist/technician with real-time information recorded by the CPC and allowing them to react to 
the observations being made. The instrument status table is placed below the chart which allows the user to easily check on the health of the instrument.

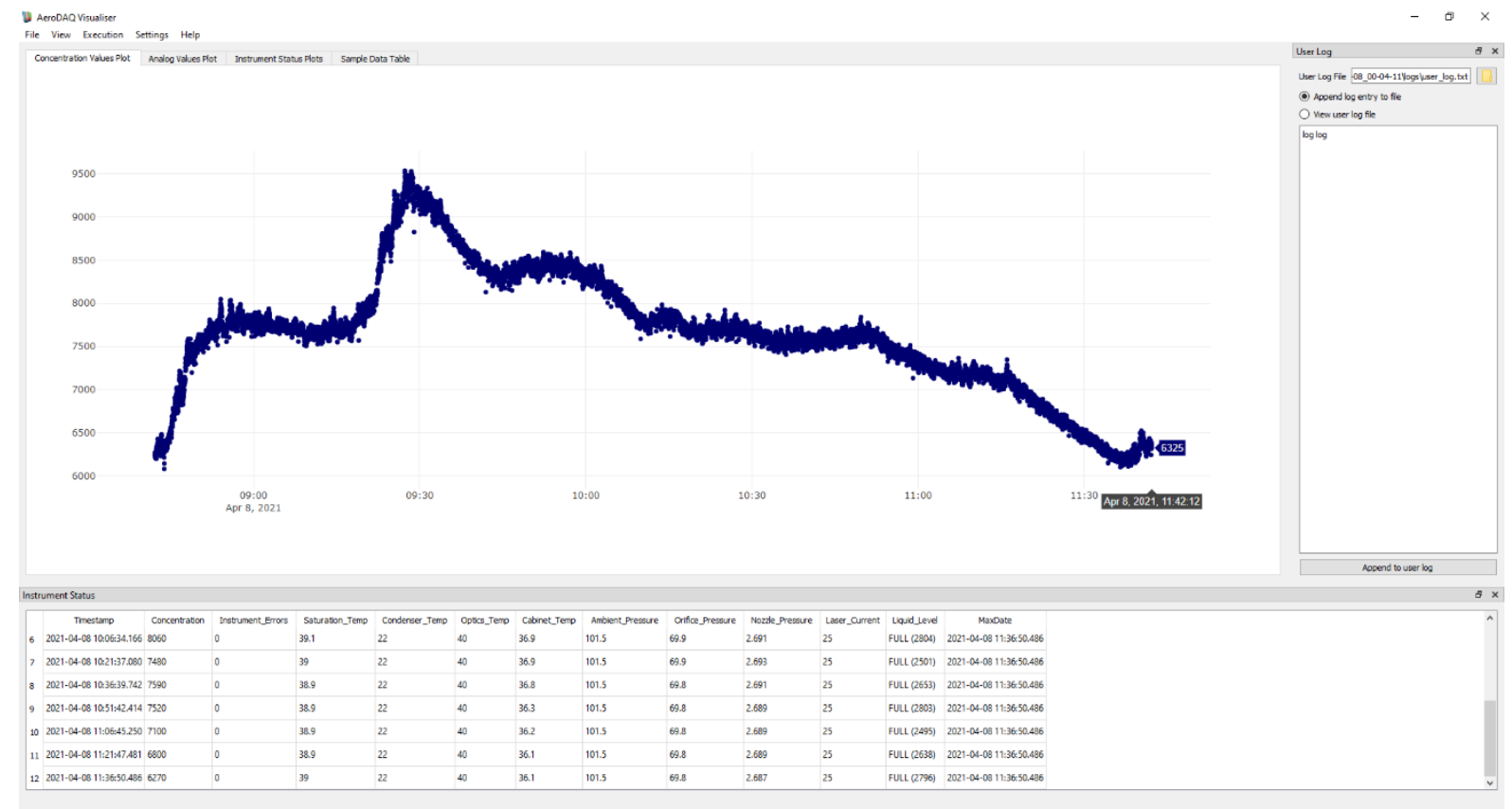

Figure 6. Concentration values chart showing concentration values for the previous 3 hours

To show instrument status over time, various instrument status charts are provided. Figure 7 shows the various charts that display instrument status temperature reports which help the user check on temporal variations in instrument status.

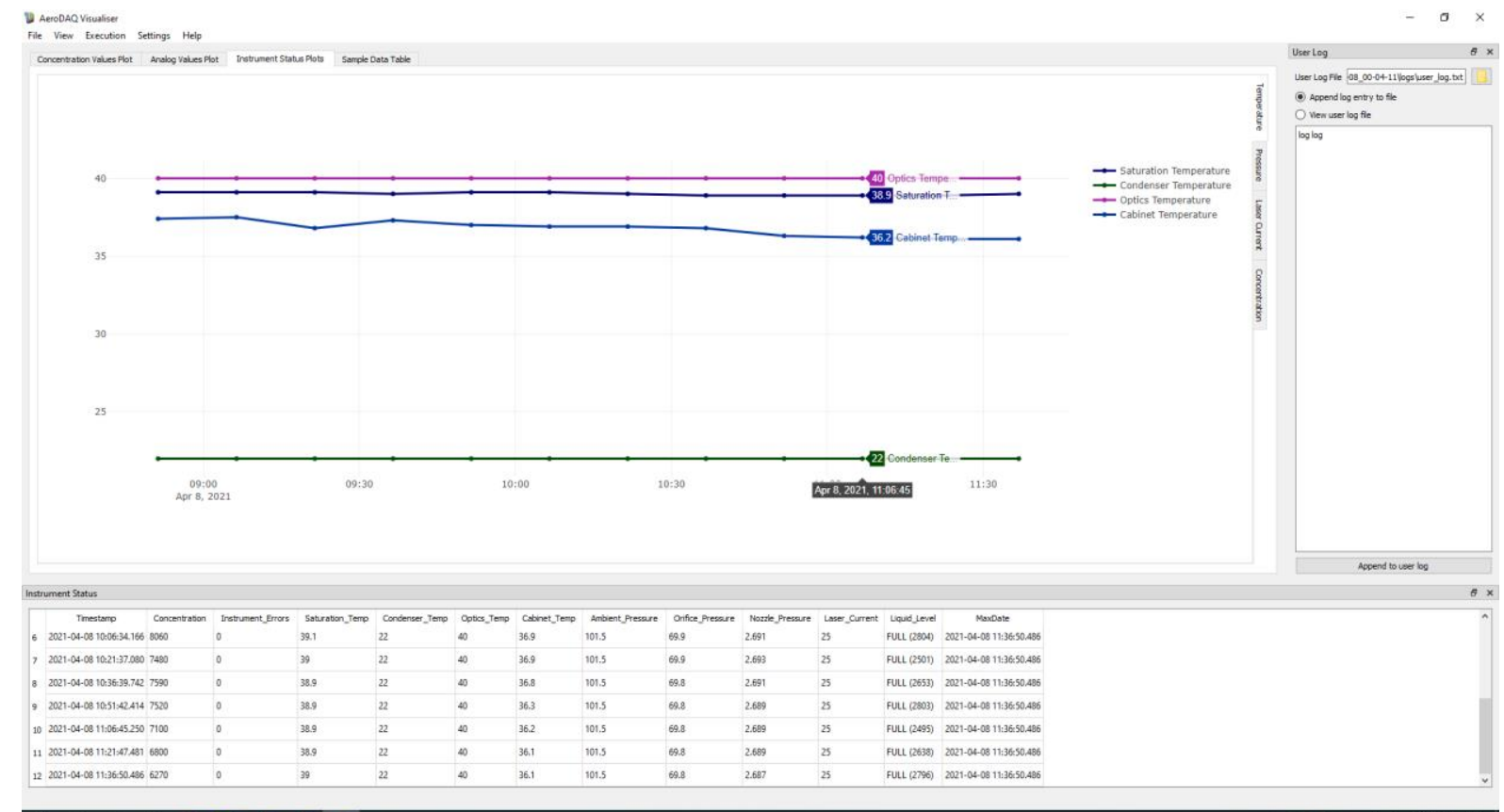

Figure 7. Instrument status - temperature values chart 
Retrospective analysis is made easier since all acquired data is stored in the form of CSV files and in a database. A new folder is created at midnight each day to store all acquired data, which is stored in the form of CSV files and in a database negating the need for laborious manual porting of data from a non-standard format.

The improvements to the workflow created by this software are significant but are largely qualitative user experience improvements which are difficult to quantify. Users report significant time savings by not having to manually deal with the issues related with the manufacturer's software (e.g., problems with exporting, timestamping and inter-compatibility issues), as well as clear advantages in identifying scientifically interesting phenomena in real-time by being able to interact with live data.

\section{CONCLUSION}

AeroDAQ is a collection of lightweight, portable, and reliable applications that manage data acquisition from the instrument, data storage and data visualisation. The AeroDAQ data collection and visualiser applications help increase the productivity of research scientists and technicians by providing a more reliable data acquisition and storage system, providing real-time visualisation of both aerosol and instrument parameter data, and storing the collected aerosol and instrument data in CSV files and a database thereby making it easier to export and use for retrospective analysis. AeroDAQ was developed using the Workspace scientific workflow engine. Workspace ensured that highly modular and reusable elements (operations, data types and widgets) were created. The Workspace editor facilitates testing of operations with inspection of often complex inputs and outputs using suitable input and display widgets. The Workspace editor also enabled the AeroDAQ workflows to be developed and tested prior to the creation of a GUI. Tailoring the user interface took a few internal and external iterations to optimise usability. Using Workspace increases the productivity of the research scientists and technicians by providing them with the ability to visualise their data in real-time, allowing them to quickly identify instrument issues. Workspace also facilitates the fast creation of applications to reliably collect, store and display data received from the CPC.

\section{REFERENCES}

- $\quad$ Bolger, M., Cleary, P. W., Hetherton, L., Rucinski, C., Thomas, D., \& Watkins, D. (2015). Workspace: Scientific Workflows and Applications for multiple Environments. Presented at the eResearch Australasia.

- Watkins, D. Thomas, L. Hetherton, M. Bolger, P.W. Cleary Workspace - a Scientific Workflow System for enabling Research Impact presented at 22nd International Congress on Modelling and Simulation, Hobart, Tasmania, Dec 3 to Dec 82017

- $\quad$ P. W. Cleary, D. Watkins, L. Hetherton, M. Bolger, D. Thomas Opportunities for workflow tools to improve translation of research into impact presented at 22nd International Congress on Modelling and Simulation (MODSIM) 2017

- $\quad$ R. C. Z. Cohen, S. M. Harrison, P. W. Cleary, M. Bolger Dive Mechanic: Bringing 3D virtual experimentation to elite level diving using the Workspace workflow engine presented at 22nd International Congress on Modelling and Simulation (MODSIM) 2017

- Anthony B. Murphy, David G. Thomas A computational model of arc welding - from a research tool to a software product presented at 22nd International Congress on Modelling and Simulation (MODSIM) 2017

- Condensation Particle Counter model 3772/3771 Operation and Service Manual and references within https://www.kenelec.com.au/wp-content/uploads/2016/06/TSI_3771_3772_Manual.pdf

- Ultrafine Condensation Particle Counter model 3776 Operation and Service Manual and references within - https://www.kenelec.com.au/wpcontent/uploads/2016/06/TSI 3776_Ultrafine_Condensation_Particle_Counter_Manual.pdf 